\title{
The analyses of the light curves of magnetic CP stars by advanced PCA methods
}

\author{
Z. Mikulášek ${ }^{1}$, J. Zverko ${ }^{2}$, J. Žižňovský ${ }^{2}$ and J. Janík ${ }^{1}$ \\ ${ }^{1}$ Institute of Theoretical Physics and Astrophysics, Faculty of Science, Masaryk University, \\ Kotlářská 2, 61137 Brno, Czech Republic \\ email: mikulas@ics.muni.cz, honza@physics.muni.cz \\ ${ }^{2}$ Astronomical Institute of Slovak Academy of Science, 05960 Tatranská Lomnica, \\ Slovak Republic, \\ email: zverko@ta3.sk, ziga@ta3.sk
}

\begin{abstract}
We present a new method of phenomenologically modelling light curves of variable stars using Principal Component Analysis techniques that provide a realistic description of the variability with a minimum of free parameters. Examples of this method are demonstrated for the magnetic CP stars HD 90044 and HD 125248.
\end{abstract}

Keywords. Methods: data analysis, methods: statistical, stars: chemically peculiar, stars: individual: (HD 90044, HD 125248), stars: magnetic fields, stars: variables: other, techniques: photometric

\section{Introduction}

A frequent problem in the study of variable stars is how to provide a quantitative description of the observed light curves taken, usually in several colors of various photometric systems. This can be optimally solved by applying Principal Component Analysis (PCA) techniques (Jollife 2002 ). PCA is a multivariate procedure suitable for constructing linear transformation of variables so that the new variables are uncorrelated with each other and arranged in the order of decreasing variance. PCA reduces the redundancy in the data by finding a new set of orthonormal axes in $Q$-space such that the projection of the data vector points is maximized. The projection on the remaining axes (components) maximizes the residual variances. As PCA does not assume any physical model or a model of an expected behavior of the object studied, it may be an effective tool for revealing hidden relationships between data and optimally reduces the dimensions of the problem.

\section{Light curves and an improvement of the period of HD 125248}

HD 125248 (HR 5353, CS Vir) (spectral type A9pSrEuCr) is a well-known magnetic, spectroscopic and photometric variable and was the first star for which the oblique rotator model was proposed (Stibbs 1950). The photometric observations of this cool magnetic CP star are considerable: Stibbs (1950) instrumental color (blue region), Maitzen \& Rakosch (1970), Maitzen \& Moffat (1972) 11-color photometry, Wolff \& Wolff (1971), Pyper \& Adelman (1985) uvby, Catalano et al. (1992) uvbyJHK, and ESA (1997) Hp.

Most of these measurements are depicted in Fig. 1, where the phase is calculated using the ephemeris: $M_{0}=24331103.95, P=9.295545 \mathrm{~d}$ which Leone \& Catanzaro (2001) derived from Stibbs, $B$ and $v$ data. Using PCA we concluded that the observed light variations in all colors can be suitably well represented by the linear combination of two 


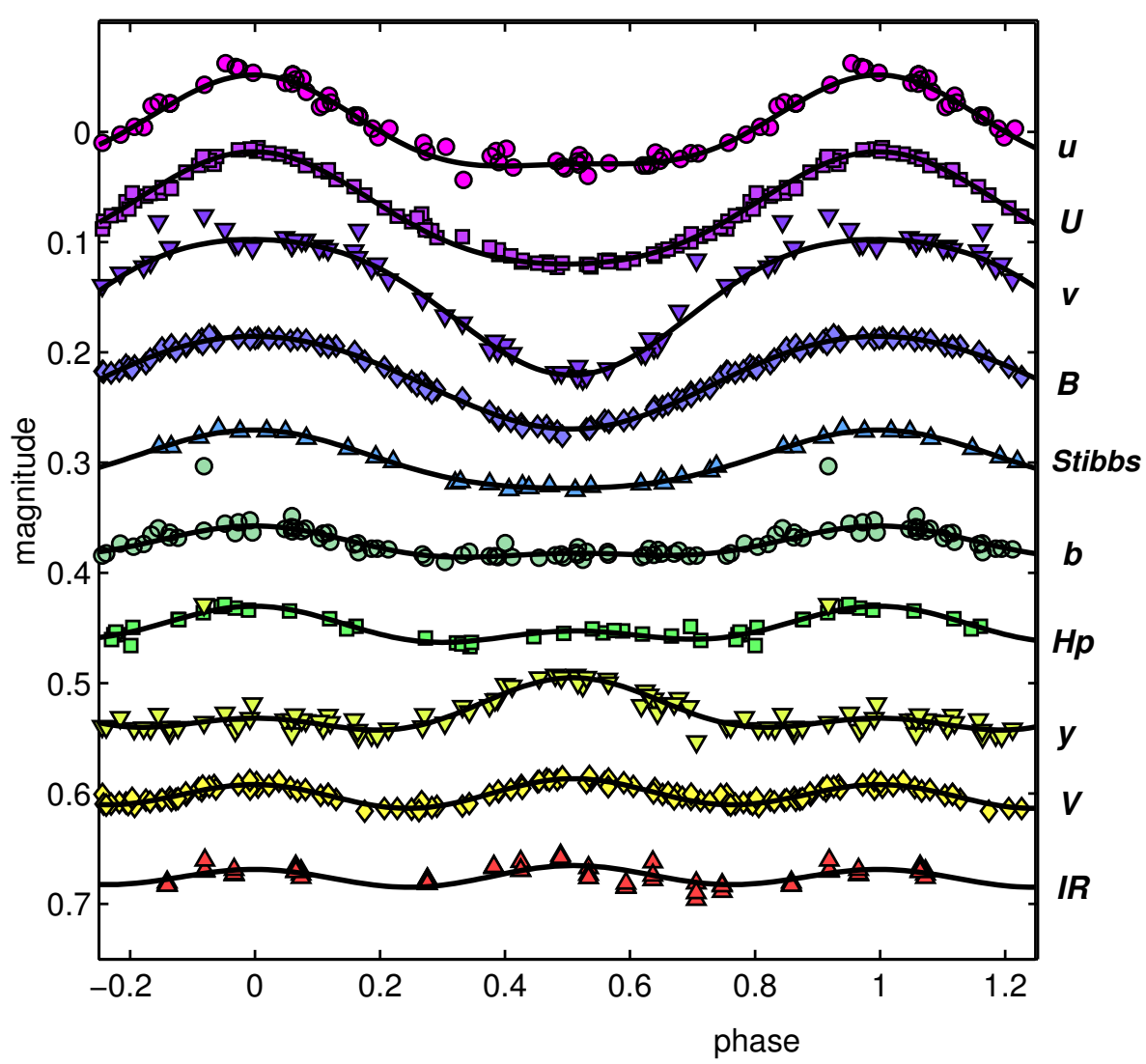

Figure 1. Light variations of HD 125248 in various colors plotted versus phase according to the ephemeris of Leone \& Catanzaro (2001). The IR color contains observations in $J, H$, and $K$ of the extended Johnson photometric system, and Stibbs (1950) observations have an effective wavelength of $455 \mathrm{~nm}$.

mutually orthogonal normalized light curves $F_{1}(\varphi) a n d F_{2}(\varphi)$ displayed on Fig. 2a.

$$
m(\varphi, c)=\overline{m(c)}+A_{1}(c) F_{1}(\varphi)+A_{2}(c) F_{2}(\varphi) .
$$

The basic function $F_{1}(\varphi)$ is described by 3 parameters (the function is normalized), the function $F_{2}(\varphi)$ is described by 2 parameters (the function is normalized and orthogonal to $\left.F_{1}(\varphi)\right)$. There are altogether five parameters.

The dependence of both semiamplitudes of HD 125248 on the wavelength is represented by a more or less smooth function what allows a plausible prediction of the variation in any color in the optical region. Note the striking dissimilarity between light curves in $V$ and $y$ and the apparent resemblance between the infrared and the $V$ light curves noticed by Catalano et al. (1992). The PCA approach decreases the parameters necessary to the describe the light curves. The standard approach with Fourier decomposition up to the first harmonic requires 81 free parameters while PCA only 50 . This saving comes in useful, e.g., when one wants to improve the ephemeris of the star.

Deutsch (1947) found the spectroscopic variability of HD 125248 with a period of 9.295 d. It was identified with the rotational period and has been improved using various type of variations. Babcock (1958) from his magnetic measurements inferred a period of 9.2954 days, Renson (1975) using light variations found 9.29541(7) d, Blanco et al. (1978) 

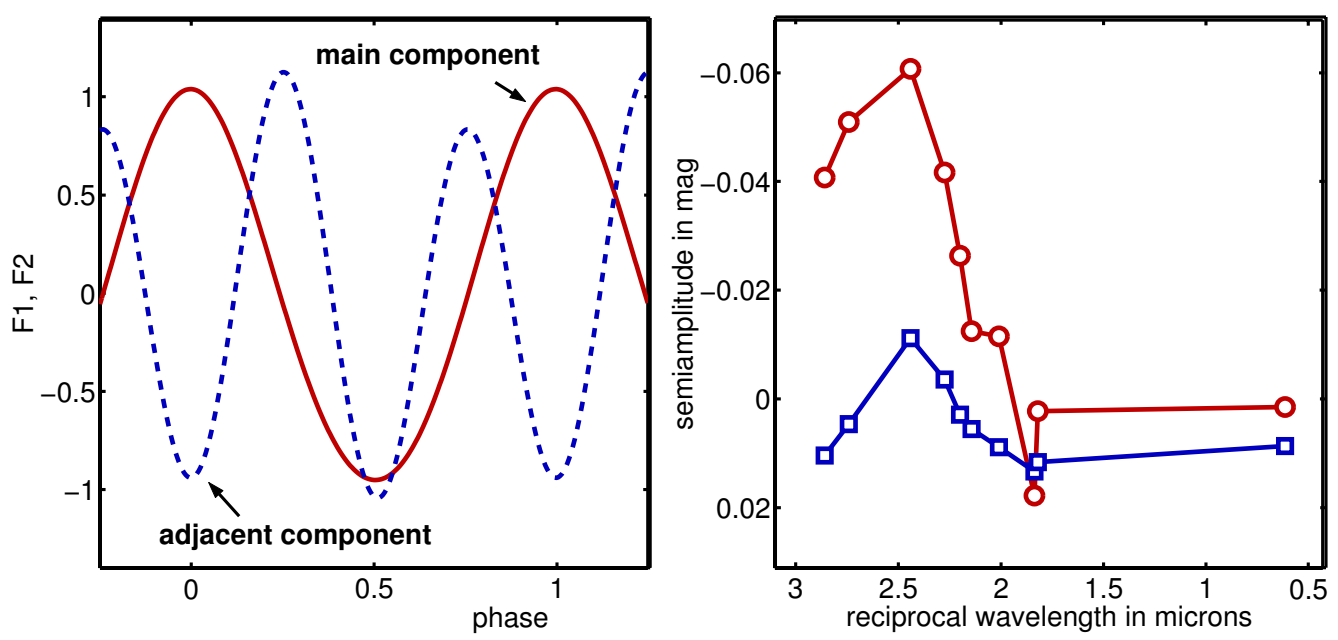

Figure 2. a) The phase diagram of the main and adjacent basic function for HD 125248; b) The dependence of its semiamplitudes on the wavelength. The uncertainties of particular points are smaller than the dimension of their markers.

from their $U B V$ photometry elicited a somewhat controversal value $9.29477(5) \mathrm{d}$ as well as Catalano et al. (1992) 9.29571(18)d, and Hipparcos 9.2870 d (ESA 1997). The period by Leone \& Catanzaro (2001) $9.29545 \mathrm{~d}$ has already been mentioned.

We improved the period of HD 125248 by using all available photometry, 592 observations over 43 years (1710 revolutions) obtained by seven authors in six photometric systems and 13 colors. As the shapes of the light curves in different colors can be dissimilar (see Fig. 1) it is not possible to transform easily measurements in the various colors to one scale. Assuming every light curve can be expressed as the linear combination of two basic functions (Eq. 1) gave the following results:

$$
J D(\mathrm{MMC})=2440698.313(12)+9.295450(30) \cdot(E-817) .
$$

The new ephemeris resembles that of Leone \& Catanzaro (2001), its mathematical background is explicitly defined, and the uncertainties are given. The zero phase corresponds to the maximum of the main principal component. The epoch is similar to that of Leone \& Catanzaro (2001). All the data used were weighted according to their uncertainties and the program employs robust procedures to eliminate the influence of outliers (Mikulášek et al. 2003). The solution is an iterative process which converges relatively rapidly.

\section{Light curves and improvement of the period of HD 90044}

HD 90044 (HR 4082, 25 Sex, SS Sex), was classified as B9p Si(Sr,Cr) by Cowley et al. (1969). Its spectrum variability was discovered by Bonsack (1974), who found variations of the Sr II and Ca II lines. Light variability was studied by Manfroid \& Renson (1983) who found a period of 4.37(4) d. During 1982-88 HD 90044 was observed in uvby as part of the Long-Term Photometry of Variable Program at ESO (Manfroid et al. 1991, Sterken et al. 1993). Renson \& Manfroid (1991) derived from them and their own observations the following ephemeris: $M_{0}(\min )=2445659.00 ; P=4.37900(4) \mathrm{d}$.

It was used by Adelman (1997) whose 109 uvby measurements were obtained in 199295. Catalano \& Leone (1993) obtained 12 uvby observations and found: $P=4.37894(15) \mathrm{d}$. 


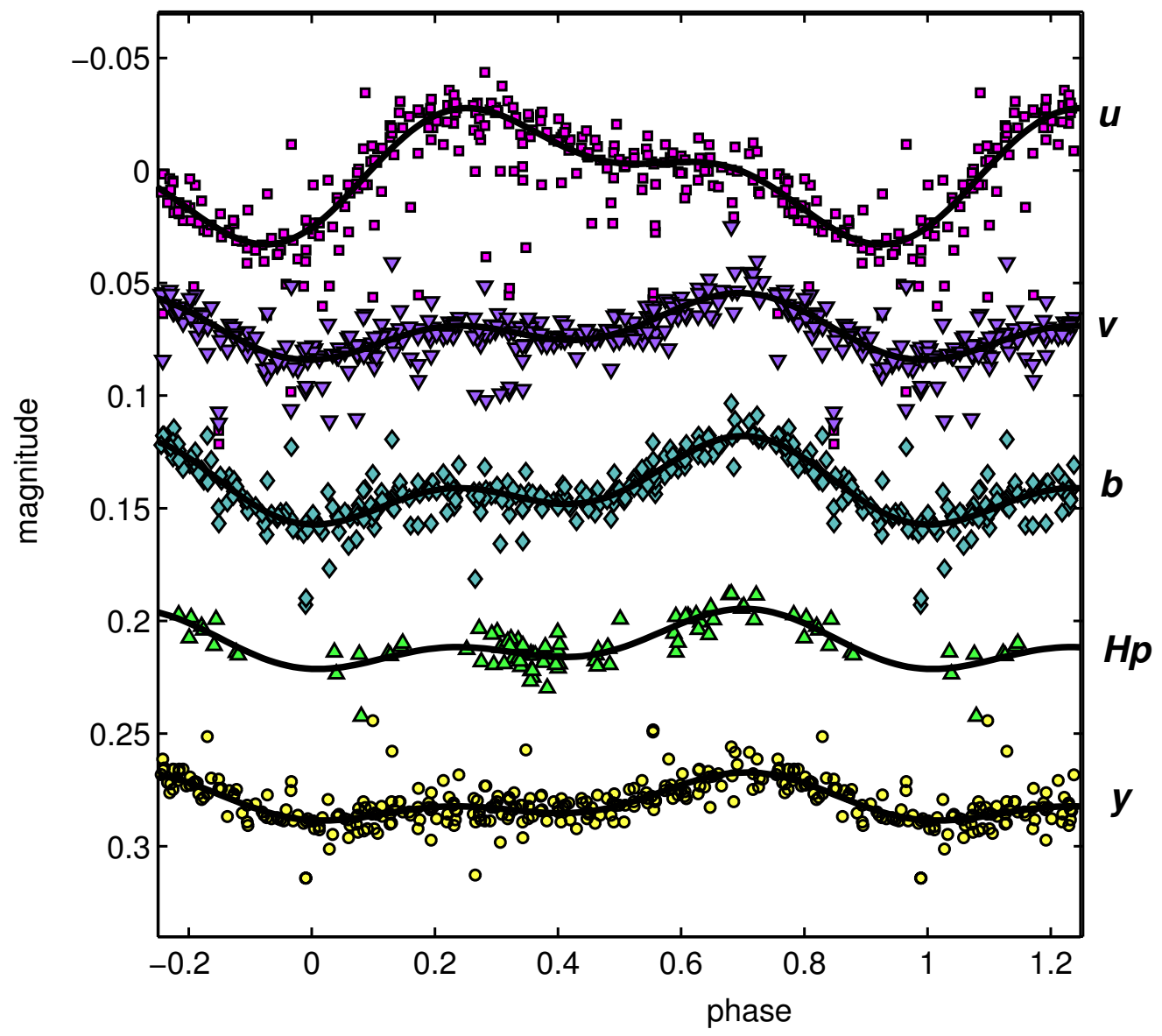

Figure 3. Light variations of HD 90044 in uvby and $H p$ colors.

The star was observed by Hipparcos ESA (1997) (83 measurements). Catalano \& Leone (1998) found single wave light variations in the near infrared.

Our ephemeris based on the 1091 individual measurements in 5 colors covering 12 years (= 1034 cycles) was found by our method. The beginning is the phase of the main principal function $\left(F_{1}(\varphi)\right)$ minimum.

$$
J D(\text { main comp. min. })=2448006.454(8)+4.378967(22) \cdot E .
$$

The light minimum in $u$ occurs at the phase -0.0076 , in $v$ at -0.006 , in $b$ at 0.005 , in $H p$ at 0.010 and in $y$ at 0.019 . See Fig. 3. The weighted standard deviation of the fit is 0.0054 mag, the worst data are in $u$ photometry which comprise a huge fraction of outliers.

\section{Conclusions}

The method based on the PCA approach is a very efficient tool of the data processing, but unfortunately it is unable to perform a real physical or geometrical modelling of the variability of a particular variable object. Nevertheless, for its simplicity and consistency it should become a useful aide for those dealing with the classification and diagnostics of variable stars. The details of our method will be described in future papers. 

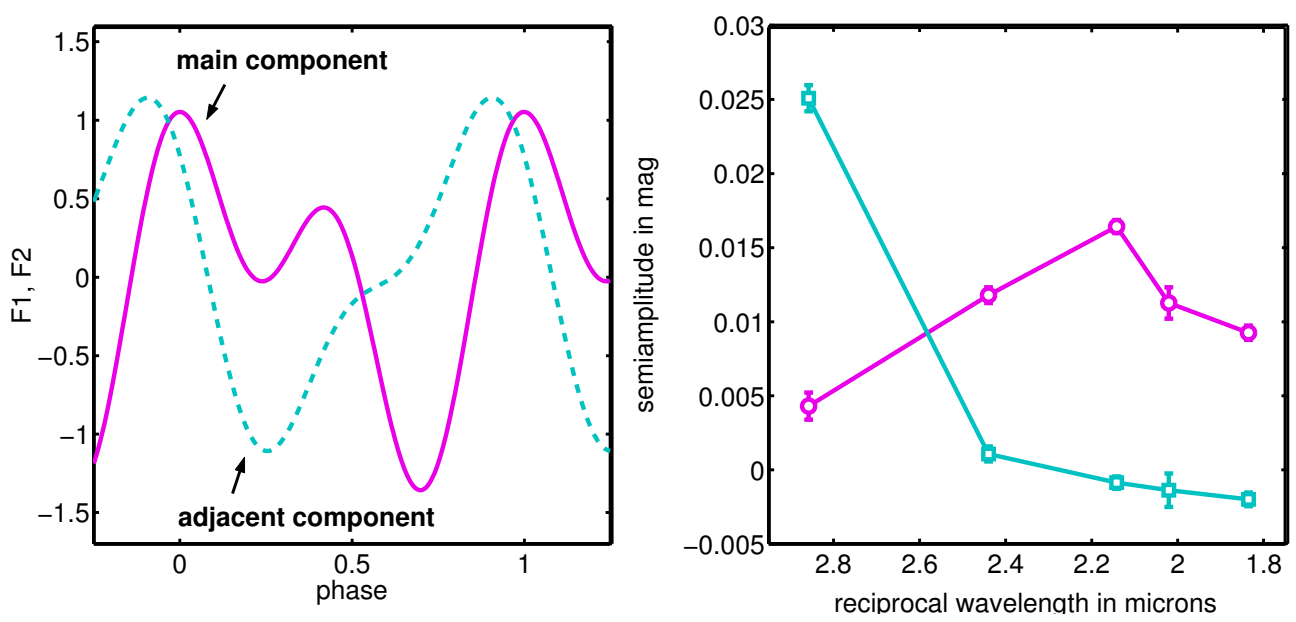

Figure 4. a) Phase diagram of the main and adjacent basic function for HD 90044; b) The dependence of its semiamplitudes on the wavelength.

\section{Acknowledgements}

This work was supported by the grants: GA ČR 205/02/0445, 205/04/1267 and Bilateral Czech-Slovak Co-operation in Research. ZM is grateful to the IAU for a travel grant.

\section{References}

Adelman, S. J. 1997, A\&BS 122, 249

Babcock, H. W. 1951, ApJ 114, 1

Blanco, C., Catalano, F. A., \& Strazulla, G. 1978, A\& $A S$ 31, 205

Bonsack, W.K. 1974, PASP 86, 408

Catalano, F. A., Kroll, R., \& Leone, F. 1992, A\&̛A 263, 203

Catalano, F. A., \& Leone, F. 1993, A\&SAS 100, 319

Catalano, F. A., Leone, F., \& Kroll, R. 1998, A\&AS 131, 63

Catalano, F. A., \& Renson, P. 1998, A\&AS 127, 421

Cowley, A., Cowley, C., Jascheks, M. \& C. 1969, AJ 74, 375

Deutsch, A. J. 1947, ApJ 105, 283

ESA 1997, The Tycho Catalogues, ESA, SP-1200

Jollife, I. T. 2002, Principal Component Analysis, Springer Verlag; 2nd edition

Leone, F., \& Catanzaro, G. 2001, A\&A 365, 118

Maitzen, H. M., \& Rakosch, K.D. 1970, A\&A 7, 10

Maitzen, H. M., \& Moffat, A. F. J. 1972, A\&A 16, 385

Manfroid, J., \& Renson, P. 1983, A\&AS 51, 267

Manfroid, J., Sterken, C., Bruch, A., et al. 1991, AESAS 87, 481

Mikulášek, Z., Žižňovský, J., Zverko, J., \& Polosukhina, N. S. 2003, Contr. Astron. Obs. Sk. Pleso 33, 29

Pyper, D. M., \& Adelman, S. J. 1985, A\&̈AS 59, 369

Renson, P. 1975, F.N.R.S. Bruxelles Gr. de C.Phys. Chim. Geophys. Geol., 19

Renson, P. \& Catalano, F. A. 2001, A\&A 378, 113

Renson, P. \& Manfroid, J. 1991, cited in: Catalano, F. A., Renson, P. \& Leone, F. 1993, A\& AS 98, 269

Sterken, C., Manfroid, J., Anton, K., et al. 1993, A\&AS 102, 79

Stibbs, D. W. N. 1950, MNRAS 110, 395

Wolff, S. C., \& Wolff, R. J. 1971, AJ 76, 422 